\title{
4-Element Concentric Pentagonal Slot-Line-Based Ultra-Wide Tuning Frequency Reconfigurable MIMO Antenna System
}

\author{
Rifaqat Hussain, Mohammad S. Sharawi and Atif Shamim
}

\begin{abstract}
In this paper, a novel, compact, ultra-wideband tuning, concentric pentagonal slot-line-based frequency reconfigurable multipleinput-multiple-output (MIMO) antenna system is presented. It consists of a 4-element antenna design on a commercially available FR-4 substrate with dimensions $60 \times 120 \times 1.56 \mathrm{~mm}^{3}$. All the antenna elements are planar in structure that are etched out from ground (GND) plane. Frequency reconfigurability is achieved using varactor diodes by reactively loading the antennas. Each capacitance value of the varactor diode results in triband operation. Proper capacitive reactance loading enables the antenna to resonate over a wide frequency band. The proposed MIMO antenna system is tuned over an ultra-wide frequency band and a smooth variation of the resonance frequencies is observed from 1.32 1.49 $\mathrm{GHz}$ and 1.75 5.2 GHz. Moreover, the unique feature of the proposed antenna system is the effectiveness of reactive loading the slot over all the resonating bands. The proposed antenna system is compact and suitable to be used in wireless handheld devices and mobile terminals for cognitive radio (CR) applications. The envelope correlation coefficient (ECC) did not exceed 0.186 in the entire operating band of the MIMO antenna operation. The maximum measured gain of the MIMO antenna is 4.5 dBi with maximum efficiency of $81 \%$.
\end{abstract}

Keywords: cognitive radio, MIMO, reconfigurable antennas, ultrawide tuning

\section{INTRODUCTION}

The tremendous growth in modern wireless communication technology requires high system throughput and efficient spectrum utilization. New standards and services are continuously added to wireless devices. To design an antenna system that covers multiple wireless standards, meet high data rate requirement and efficiently utilize the spectrum resources is a formidable challenge for antenna designers. The aforementioned challenges can be addressed by utilizing multiple-input-multiple-output (MIMO) systems and frequency agile antennas in cognitive radio (CR) platforms. CR are being developed to effectively utilize spectrum resources [1]. It is highly desirable and key factor to use wideband tuning frequency reconfigurable MIMO antenna systems in second generation CR based applications [2].

Several frequency reconfigurable wideband tuning antennas were reported in literature [3]-[8]. In [3], a dual-mode frequency reconfigurable slot-based antenna was presented. The substrate used was RO4003 with board dimensions of $150 \times 110 \times 0.5 \mathrm{~mm}^{2}$. The frequency sweep obtained was $1.3 \sim 2.67 \mathrm{GHz}$ by applying reverse bias voltage for two different sets of biasing voltages. In [4], a printed inverted Fshaped antenna (PIFA) was presented covering frequency bands from $0.778 \sim 2.896 \mathrm{GHz}$. The elevated PIFA had dimensions of $40 \times 5 \times 7$ $\mathrm{mm}^{3}$ with ground (GND) plan size of $100 \times 40 \mathrm{~mm}^{2}$.

In [5], an electrically small frequency reconfigurable PIFA antenna was presented covering low frequency bands from $0.457 \sim 0.894$ GHz. The elevated PIFA had dimensions of $50 \times 50 \times 15 \mathrm{~mm}^{3}$ on GND plan size of $200 \times 200 \mathrm{~mm}^{2}$. In [7], a ultra-wideband and dual-band half annular-slot based frequency reconfigurable antenna was presented. The proposed design was covering three widebands:

R. Hussain and M. S. Sharawi are with the Electrical Engineering Department, King Fahd University for Petroleum and Minerals (KFUPM), Dhahran, 31261 Saudi Arabia, Email: \{rifaqat, msharawi\}@kfupm.edu.sa. A. Shamim is with the Computer, Electrical and Mathematical Science and Engineering Division, King Abdullah University (KAUST) of Science and Technology, Thuwal 23955-6900, Saudi Arabia, Email:atif.shamim@kaust.edu.sa
$3.48 \sim 5.5,5.18 \sim 7.35$, and $6.06 \sim 8.05 \mathrm{GHz}$ while in dual band it covered $2.5 \sim 3.22 \mathrm{GHz}$ for the first resonance and $4.36 \sim 6.58 \mathrm{GHz}$ for the second resonance. The annular slot had a radius of $10.5 \mathrm{~mm}$ on a GND plan area of $32 \times 35 \mathrm{~mm}^{3}$.

Several MIMO reconfigurable antenna designs were reported in literature for CR applications with wide tuning bands [9]-[15]. In [12], a 2-element frequency agile MIMO slot antenna was presented. The given antenna was covering several frequency band: $2.4 \sim 2.5$ $\mathrm{GHz}, 4.9 \sim 5.725 \mathrm{GHz}$ and $4.9 \sim 5.725 \mathrm{GHz}$. The single element diemension used were $3 \times 5 \mathrm{~mm}^{2}$ with GND plan size of $46 \times 20 \times 1.6$ $\mathrm{mm}^{3}$. In [13], a 2-element PIFA was operating over a frequency band from $2.3 \sim 2.4 \mathrm{GHz}, 2.5 \sim 2.7 \mathrm{GHz}$, and 3.4 3.6 GHz. The single element dimensions used were $10 \times 10 \times 6.4 \mathrm{~mm}^{3}$. In [14], 2element MIMO antenna was presented to cover a frequency band from $1.71 \sim 1.88 \mathrm{GHz}$ and $2.5 \sim 2.7 \mathrm{GHz}$. The antenna was fabricated on substrate board of dimensions $150 \times 150 \mathrm{~mm}^{2}$. Similarly, a 2element MIMO PIFA was presented in [15] with frequency band coverage from $2.1 \sim 2.9 \mathrm{GHz}$. The antenna dimension used were $16 \times 33 \times 5 \mathrm{~mm}^{3}$.

In [16], simple rectangular slot antenna was presented with wide tuning of operating bands. The given antenna was made reconfigurable using a combination of PIN and varactor diodes. The wide continuous band covered was from $0.42 \sim 1.48 \mathrm{GHz}$. The single element slot antenna had dimensions of $25 \times 1.5 \times 1.56 \mathrm{~mm}^{3}$ on substrate board of size of $35 \times 20 \mathrm{~mm}^{2}$. In [17]-[23], various slot-based frequency reconfigurable antennas were presented. The slot shapes studied were rectangualr, annular, rectangular, rotated $\mathrm{T}$ along with $\mathrm{U}$-shape. The performance of the proposed antenna design was compared interms of various antenna parameters, analyzed completely and the results are presented in Table II.

In this work, a 4-element concentric pentagonal slot-line-based MIMO antenna system is presented. The proposed MIMO antenna system is made reconfigurable using two varactor diodes per antenna element. The proposed design is a tri-band antenna system with an ultra-wide tuning capability. All the resonant bands were made reconfigurable and thus covering a very wide-tuning-band from $1.32 \sim 1.49 \mathrm{GHz}$ and $1.75 \sim 5.2 \mathrm{GHz}$. The resonance frequencies were smoothly varied over the entire bands. The proposed MIMO antenna system covers all major wireless standard band. The distinguishing features of the proposed design are its compact size, planar structure and its ultra-wide tuning across several frequency bands. Moreover, the proposed antenna design is also analyzed for MIMO performance metrics and good results are obtained. The proposed CR antenna system was realized on substrate volume of $60 \times 120 \times 1.56 \mathrm{~mm}^{3}$. The proposed design is suitable to be used as a front-end in wireless handheld devices and mobile terminals for second generation CR platforms.

The of the paper is organized as follows. Sections II describes the antenna system design. Simulation and measurement results for the MIMO antenna system are given in Section III. The conclusions are provided in Section IV. 


\section{Design Details of the MiMO Antenna System}

The geometry of the proposed antenna design is shown in Fig. 1. It consists of 4-element concentric slot-line-based frequency agile MIMO antenna system. The antenna was fabricated on commercially available FR-4 substrate with a relative permitivity $\left(\epsilon_{r}\right)$ of 4.4 and loss tangent $(\delta)$ of 0.002 . The fabricated model of the proposed antenna design is shown in Fig. 2. In the proposed design, each antenna element consisted of dual concentric pentagonal slot-line etched out of the GND. The pentagonal shape was used because it gives wide and multi-band response when designed properly. Good impedance matching can be obtained using this shape compared to others (i.e. circular or rectangular). The length of each side of the outer and inner pentagonal slot-lines were $14.2 \mathrm{~mm}$ and $8.3 \mathrm{~mm}$, respectively. The dimensions and placement of both the pentagonal slot-line were optimized to get the good MIMO performance of the proposed design. The term good MIMO performance means to ensure proper MIMO antenna operations via providing good metrics performance. The critical parameters (isolation between ports, envelope correlation coefficient (ECC) and total active reflection coefficient (TARC)) used for MIMO evaluation are carefully assessed and the design is optimized accordingly. Ideally high isolation, low ECC and robust TARC values are required for good MIMO operation. However, in real designs, $10 \mathrm{~dB}$ isolation is sufficient for most of the communication standards, ECC values less 0.5 are acceptable and TARC values with quite robust bandwidth are required.

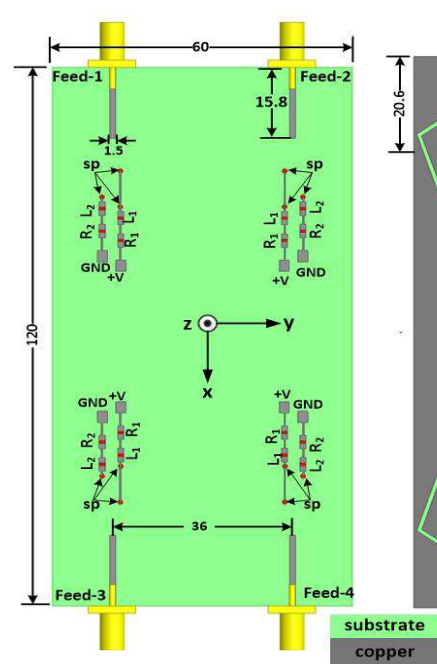

(a)

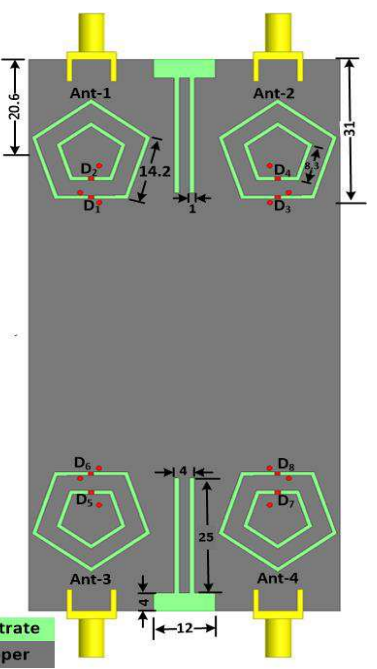

(b)
Figure 1: Proposed MIMO antennas system for CR platform (a) Top view (b) Bottom view - All dimensions are in millimeters (mm).

The proposed MIMO antenna design was fabricated using an LPKF S103 machine. Fig. 2(a) shows the top view of the fabricated design with the varactor diode biasing circuitry and feeding lines. Fig. 2(b) shows the GND plane having 4-element pentagonal shaped concentric slot-line that were etched out from the GND plane.

The design procedure of the proposed concentric pentagonal slotline-based antenna was started with a single pentagonal slot-line antenna fed with $50 \Omega$ microstrip line. The antenna dimensions of the single band outer pentagonal slot-line were optimized to make it resonate at $3 \mathrm{GHz}$ without reactive loading of the slot. For the optimized design, an internal pentagonal slot-line was introduced, with the same feeding line, resulting in a tri-band antenna. In the proposed design, the single pentagonal shape exhibited good input impedance matching that resulted in a wide sweep of resonance frequencies over a wide band by reactively loading it with good matching. On the other hand, other slot shapes will result in poor matching over a wideband, and it is quite difficult to achieve wideband frequency tuning as input impedance matching is lost after a short frequency band. The addition of a secondary slot structure (which is the replica of primary structure) also exhibited good matching at higher frequency bands and it also helped in wideband tuning. The two different size slots with different resonance bands resulted in ultra-wide-tuning operation.

To make the antenna a frequency agile one, parametric sweeps were performed to properly place the varactor diodes to effectively load both the inner and outer slots. The current positions of the varactor diodes provided the best tuning effect on the antenna resonances. Varactor diodes $D_{1}$ and $D_{2}$, were utilized to reactively load Ant-1. The varactor diode terminals were connected to a biasing circuit using two shorting pins (sp)/vias as shown in Fig. 1. All the four antenna elements (Ant-1, Ant-2, Ant-3 and Ant-4) were exactly of similar structure and were placed symmetrically on the substrate board. As the proposed design is targeting wireless handheld devices and mobile terminals, we have placed the antenna elements on the top and bottom edges of the substrate board to provide room for other component such as battery and screen. Varactor diodes used in all four antenna elements are designated as $D_{1}, D_{2}, D_{3}, D_{4}, D_{5}, D_{6}, D_{7}$ and $D_{8}$.

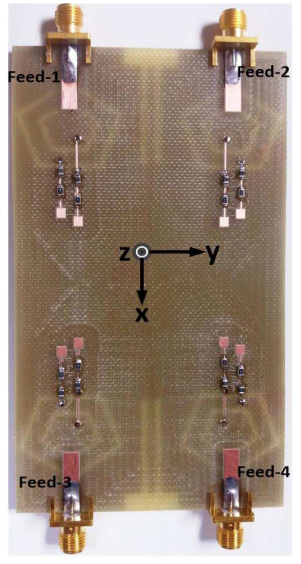

(a)

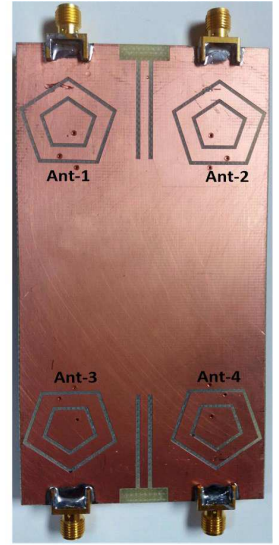

(b)
Figure 2: Fabricated prototype. (a) Top view (b) Bottom view.

A defected ground structure (DGS) was introduced between two adjacent antenna elements to minimize the mutual coupling. As the closely spaced antenna elements were exhibiting high mutual coupling at lower frequency bands while the mutual coupling was acceptable in the higher bands. It was necessary to take some corrective measures to minimize this. For this purpose, the current distribution was analyzed between adjacent antenna elements and it was observed that mostly the coupling was from the antenna's edges. The proposed DGS structure enabled the antenna to have minimal coupling even at lower frequency bands.

The varactor diode was reverse biased and different capacitance values were obtained for different reverse bias voltage levels. The circuit consists of a series combination of an RF choke of $1 \mu \mathrm{H}$ $\left(L_{1}\right.$ and $\left.L_{2}\right)$ and $2.1 \mathrm{k} \Omega$ resistors $\left(R_{1}\right.$ and $\left.R_{2}\right)$ connected to the two terminals of the varactor diode. The radiating antenna structure was isolated from power supply section using an RF choke while DC leakage current entering the antenna section was effectively blocked by the varactor diode itself. The diodes were utilized to tune the resonance frequency over a wide operating band. The varactor diodes used were SMV 1233. This varactor diode model is available in 0805 surface mount package with a standard dimensions of $2.0 \mathrm{~mm} \times 1.2$ $\mathrm{mm}$. This package is selected to ease the soldering process and due to its availability in our lab. Any other varactor diode type can be used in this design with the same capacitance variation range with different 
reverse bias voltage $(0 \sim 3.3)$ to achieve the same performance in a practical power limited design.

\section{Simulation and Measurement Results}

The proposed 4-element concentric pentagonal slot-line-based reconfigurable MIMO antenna system was modeled and simulated using $\mathrm{HFSS}^{T M}$. The S- parameters were measured using an Agilent N9918A vector network analyzer. Fabrication and measurements were performed at the Antenna and Microwave Structure Design Lab (AMSDL) at King Fahd University of Petroleum and Minerals (KFUPM). The gain patterns and efficiencies were measured at King Abdullah University of Science and Technology (KAUST), Saudi Arabia, using a SATIMO Starlab anechoic chamber.

\section{A. MIMO Antenna Scattering Parameters}

The proposed MIMO antenna system was characterized for scattering parameters. As all the antenna elements were exactly similar in structure and placed symmetrically on the substrate board, hence similar results were obtained from all the ports. For brevity, the results obtained from one port are discussed in details here. The proposed MIMO antenna system was made reconfigurable using varactor diodes. The capacitance of the diode was varied using the reverse bias voltage. The varactor diode was modeled as a variable capacitance in HFSS.

The simulated reflection coefficient curves for Ant-1 are shown in Figs. 3(a) and 3(b). The frequency range from 1 to $5.5 \mathrm{GHz}$ is divided into two ranges: 1 to $3 \mathrm{GHz}$ and 3 to 5.5 , to get better frequency resolution. The proposed design is tri-band antenna with wideband tuning capability (i.e. can sweep wide bands during reconfigurability). At a capacitance value of $0.9 \mathrm{pF}$, the antenna was resonating, with center frequencies at $4.94 \mathrm{GHz}, 2.98 \mathrm{GHz}$ and $1.46 \mathrm{GHz}$. A wide sweep of resonance frequencies was observed by varying the capacitance values from $0.9 \mathrm{pF}$ to $5.08 \mathrm{pF}$. The frequency sweep obtained in the three bands were: $1.32 \sim 1.49 \mathrm{GHz}, 1.76 \sim 3.10$ $\mathrm{GHz}$ and $3.10 \sim 5.2 \mathrm{GHz}$ with minimum operating bandwidth (BW) of $50 \mathrm{MHz}$. In other words, the proposed antenna design can be tuned to any frequency band from $1.32 \sim 1.49 \mathrm{GHz}$ and also from $1.76 \sim 5.2$ $\mathrm{GHz}$. This extremely wide band coverage is the distinguishing feature of the proposed reconfigurable MIMO antenna design.

The measured reflection coefficient curves for the MIMO antenna system are shown in Figs. 3(c) and 3(d) for frequency band from $1 \sim 3 \mathrm{GHz}$ and $3 \sim 5.5 \mathrm{GHz}$, respectively. The measured frequency bands obtained were from $1.31 \sim 1.495 \mathrm{GHz}$ and $3.05 \sim 5.5 \mathrm{GHz}$ with minimum $-6 \mathrm{~dB}$ operating $\mathrm{BW}$ of $55 \mathrm{MHz}$. The measurement results showed almost the same covered resonance frequencies as obtained in simulation and good agreement was found between the two results. The small variation in measurements were attributed to fabrication tolerances and substrate properties. The small discrepancy in measured results can be easily compensated for as we have a continuous sweep of resonance frequencies and the proposed antenna can be tuned to any desired resonance frequency.

The proposed MIMO antenna system was also evaluated for mutual coupling among various antenna elements. Initially, the closely spaced antenna elements Ant- 1 and Ant- 2 were poorly isolated as observed from the surface current density. The two antenna elements were strongly coupled on the edges, that is why the proposed DGS structure was introduced between Ant- 1 and Ant- 2 near the board edges. The simulated and measured isolation curves for closely spaced antenna elements, Ant-1 and Ant-2, are shown in Figs. 3(e) and 3(f), respectively, for Ant-1 and Ant-2. The coupling between antenna elements was high at lower frequencies while it gradually went down to lower values at higher frequency bands. Mutual couping values were more than $12 \mathrm{~dB}$ between all antenna elements at all the operating bands. The simulated and measured isolation values were also computed between Ant-1 \& Ant-3 and the worst case values observed were $13.1 \mathrm{~dB}$ and $13.3 \mathrm{~dB}$, respectively, in the entire operating band.

\section{B. Current Distribution}

For the given MIMO antenna system, the surface current distribution was observed from simulations for different resonance frequencies. It helped in understanding how the various ports were coupled and the location of maximum concentration of the surface current density. The observations were also helpful in reducing the port coupling by properly designing the DGS structure for better MIMO performance. Fig. 4(a) shows the surface current density obtained at frequency band of $1.86 \mathrm{GHz}$ without DGS structure corresponding to varactor diode capacitance $C$ equal to $5.08 \mathrm{pF}$. In this configuration, Ant- 1 was excited while all other ports were terminated with $50 \Omega$ impedance. As seen from the figure that although Ant-1 was the main radiator, Ant-2 had high current levels. Fig. 4(b) shows the surface current density obtained at same frequency band but with the DGS structure. It is clear from the figure that most of the current was around Ant-1 and radiation was mainly from its slots. The current coupling observed between Ant-1 and Ant-2 was very low and hence better isolation was obtained with DGS structure. The simulated values of isolation $\left(S_{21}\right)$ were $11.5 \mathrm{~dB}$ without DGS as shown in Fig. 4(a) while it improved to $14 \mathrm{~dB}$ as shown in Fig. 4(b).

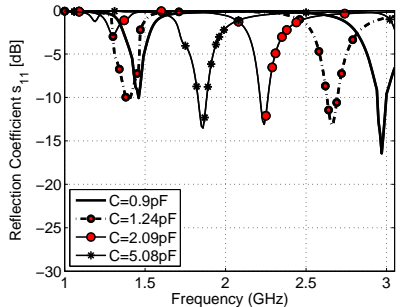

(a)

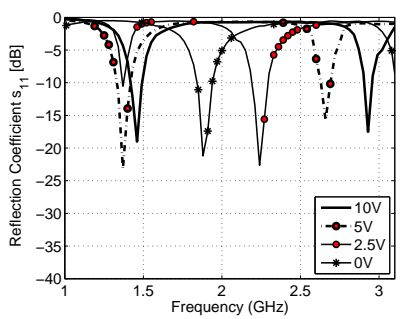

(c)

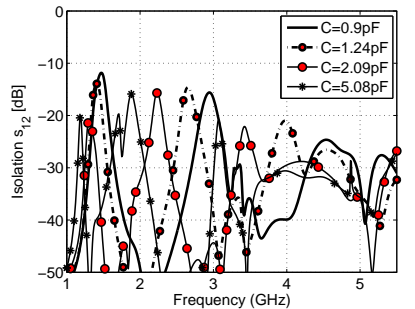

(e)

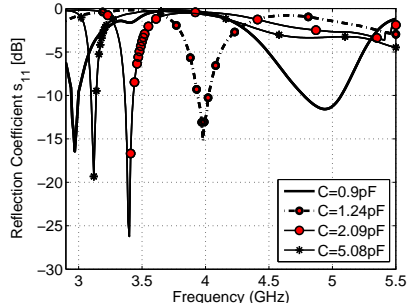

(b)

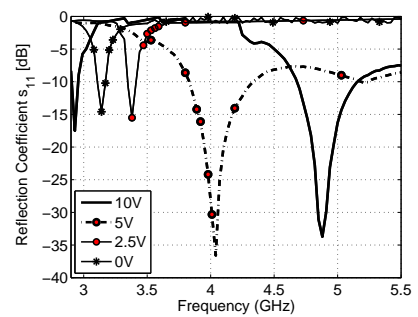

(d)

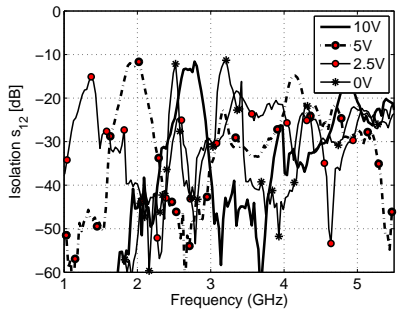

(f)
Figure 3: (a) $\left|s_{11}\right|$ - simulated (b) $\left|s_{11}\right|$ - simulated (c) $\left|s_{11}\right|$ - measured (d) $\left|s_{11}\right|$ - measured (e) $\left|s_{12}\right|$ - simulated (f) $\left|s_{12}\right|$ - measured

\section{Far Field Radiation Characteristics of the MIMO Antenna}

For the proposed reconfigurable MIMO antenna, 3-D gain patterns were computed using HFSS as shown in Fig. 5. The gain patterns are plotted for all 4-antenna elements at a frequency band of $2.98 \mathrm{GHz}$. It is clear from the given figure that all the patterns are tilted away from each other which help in reducing the field coupling between antenna 


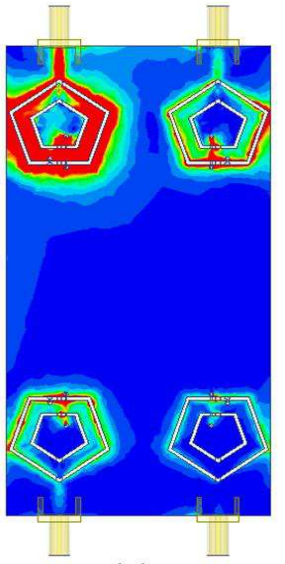

(a)

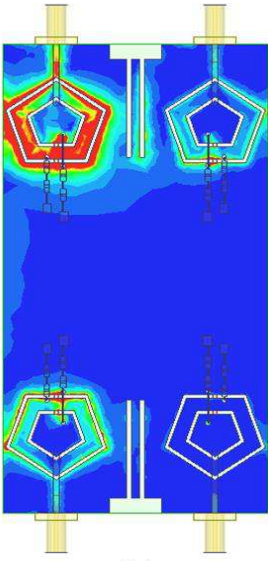

(b)
Figure 4: Surface current density at $1.86 \mathrm{GHz}(C=5.08 \mathrm{pF})$ (a) Top view (b) Bottom view

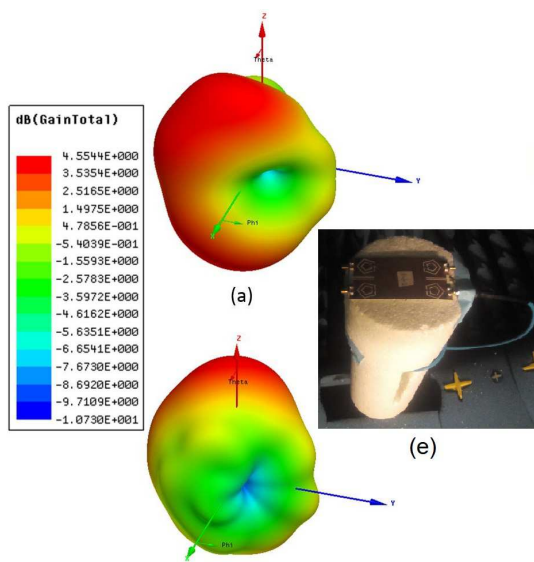

(c)

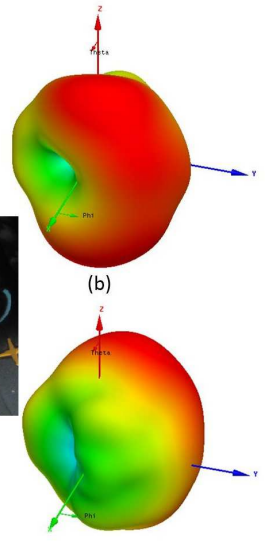

(d)
Figure 5: Simulated 3-D gain pattern at $2.98 \mathrm{GHz}(\mathrm{a})$ Ant-1 (b) Ant-2 (c) Ant-3 (d) Ant-4 (e) Radiation pattern measurement setup.

elements. This is highly desirable for improved MIMO antenna performance. All the antenna elements are structurally symmetrical however the patterns observed are non-symmetrical. All the antenna elements are placed symmetrically but they are non-symmetrical with respect to GND plan and with reference axis. The large GND plane on one side of the antenna element and the presense of an adjacent elements act as reflectors and pushes the radiated fields away from the antenna. Hence a tilt is observed in the pattern of each antenna element as shown in the 3D patterns in Fig. 5.

The pattern and efficiency measurements setup is shown in Fig. 5(e). The design was measured at KAUST using a SATIMO Starlab anechoic chamber. Each antenna element was measured for its pattern and efficiency while other ports were terminated with $50 \Omega$ impedance in an anechoic chamber. The efficiencies were measured using the gain directivity method. The measured normalized $2 \mathrm{D}$ gain patterns for frequencies at $1.86 \mathrm{GHz}$ and $2.65 \mathrm{GHz}$ are given in Figs. 6, 7 respectively. The patterns show the $\mathrm{E}_{\phi}$ and $\mathrm{E}_{\theta}$ for all elements (Ant1 to Ant-4) at $\phi=0^{\circ}$ and $\phi=90^{\circ}$. Fig. 8 shows the measured normalized 2D gain pattern for the frequency band at 5.0 GHz. Figs. 8(a) - 8(d) show $\mathrm{E}_{\phi}$ and $\mathrm{E}_{\theta}$ for all 4-antenna elements for at $\phi=0^{\circ}$ and $\phi=90^{\circ}$, respectively.

A comparison between simulated and measured peak gains and efficiencies are provided in Table I. Good agreement between simulated and measured values is observed. The simulated and measurement
Table I: Antenna efficiency and peak gain of the MIMO antenna system

\begin{tabular}{|c|c|c|c|c|}
\hline \multirow{2}{*}{$\mathrm{f}_{\text {sim }}, \mathrm{f}_{\text {mea }}(\mathrm{GHz})$} & \multicolumn{2}{|c|}{ Peak Gain $(\mathrm{dBi})$} & \multicolumn{2}{c|}{ Efficiency $(\%)$} \\
\cline { 2 - 5 } & Simulated & Measured & Simulated & Measured \\
\hline $4.94,4.88$ & 5.2 & 4.5 & 85 & 75 \\
\hline $3.98,4.04$ & 4.9 & 4.45 & 78 & 68 \\
\hline $3.4,3.38$ & 4.82 & 4.08 & 80 & 71 \\
\hline $3.12,3.14$ & 4.67 & 3.98 & 83 & 79 \\
\hline $2.98,2.98$ & 4.55 & 3.80 & 87 & 81 \\
\hline $2.65,2.66$ & 3.85 & 3.12 & 68 & 60 \\
\hline $2.24,2.45$ & 3.1 & 2.78 & 65 & 56 \\
\hline $1.86,1.88$ & 2.29 & 1.91 & 60 & 51 \\
\hline $1.46,1.47$ & 0.89 & 0.5 & 45 & 40 \\
\hline
\end{tabular}

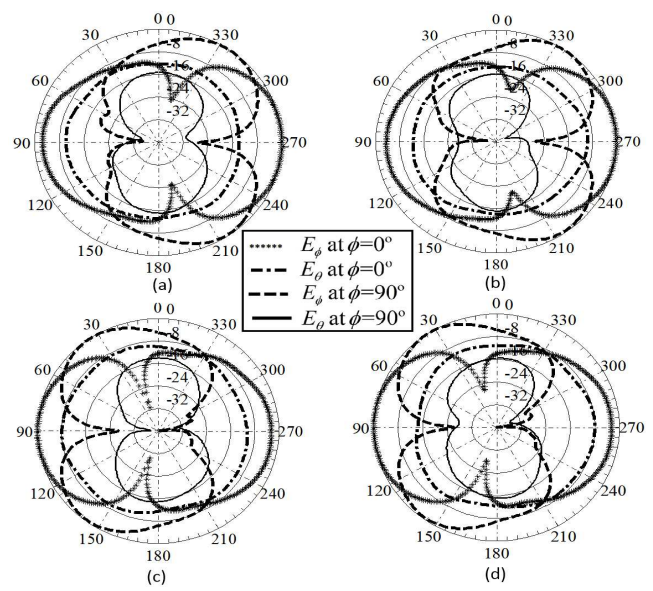

Figure 6: Measured normalized $\mathrm{E}_{\theta}$ and $\mathrm{E}_{\phi}$ at $1.86 \mathrm{GHz}$ (a) Ant-1 (b) Ant-2 (c) Ant-3 (d) Ant-4.

frequencies are designated as $f_{\text {sim }}$ and $f_{\text {mea }}$ (in GHz), respectively.

Table II compares the features of the proposed work with other wide tunable frequency reconfigurable antennas as well as other various slot-based designs. In addition, a comparison of concentric pentagonal slot-line based antenna with other most relevant slotbased frequency reconfigurable antennas [16]-[23] are also provided in details in Table II. The features of the proposed concentric pentagonal slot-line based design out-weighs other relevant designs. It has compact size, using minimum number of varactor diodes for achieving reconfigurability and provides ultra-wideband frequency sweep of resonating bands. None of the designs cited in Table II has a continuous frequency sweep except [17] but over a limited range from $1.8 \sim 2.5 \mathrm{GHz}$ with a comparable antenna dimensions. It is clear from given Table that the proposed design is one of the distinguished work of slot-based antenna design available in literature.

\section{ECC and TARC for MIMO Antenna}

The ECC is an important parameter to be considered for good MIMO performance. It is a measure of field coupling between closely spaced MIMO antenna elements and it shows how different MIMO channels are correlated with each other. ECC values were calculated using radiation patterns of the antennas as given in [24]. 
Table II: Comparison between the proposed antenna and other works.

\begin{tabular}{|c|c|c|c|c|c|c|c|}
\hline Ref. & Ant. Type & $\begin{array}{l}\text { Single elem. } \\
\text { area }\left(\mathrm{mm}^{2}\right)\end{array}$ & $\begin{array}{l}\text { GND size } \\
\left(\mathrm{mm}^{2}\right)\end{array}$ & $\begin{array}{l}\text { Freq. bands } \\
(\mathrm{GHz}) \text { covered }\end{array}$ & $\begin{array}{l}\text { Diodes } \\
\text { used? }\end{array}$ & Peak Gain (dBi) & Max Efficiency $(\% \eta)$ \\
\hline [4] & PIFA & 200 & $100 \times 40$ & $0.778 \sim 2.896$ & 2 & - & $\begin{array}{c}86.5 \% \text { @ } 0.932 \mathrm{GHz}, 91 \% \\
\text { @ } 2.136 \mathrm{GHz}\end{array}$ \\
\hline [6] & monopole & 238 & $130 \times 230$ & $0.419 \sim 0.883$ & 1 & - & $\begin{array}{c}12 \% @ 0.419 \mathrm{GHz}, 75 \% @ \\
0.880 \mathrm{GHz} \\
\end{array}$ \\
\hline$[8]$ & bow-tie & 2250 & $45 \times 50$ & $3 \sim 6$ & 2 & above 3.1 & - \\
\hline [9] & monopole & 1330 & $49 \times 55$ & $1.88 \sim 2.64$ & 2 & above 1.93 & - \\
\hline [10] & dipole & 200 & $118 \times 40$ & $\begin{array}{c}0.705 \sim 0.951, \\
1.692 \sim 2.457, \\
2.862 \sim 3.0\end{array}$ & & above -2.480 & above $65 \%$ \\
\hline [11] & PIFA & 212.5 & $60 \times 100$ & $1.610 \sim 2.71$, & 6 & above 3 & above $70 \%$ \\
\hline [16] & rect. slot & 37.6 & $35 \times 20$ & $0.42 \sim 1.48$ & 2 & above -0.4 & - \\
\hline [17] & annul. slot & 320 & $60 \times 120$ & $1.8 \sim 2.5$ & 1 & $-2.6,0.5,2.1,2.36,2.43$ & $\begin{array}{c}51 \%, 67 \%, 71.5 \%, 72 \%, \\
73 \%\end{array}$ \\
\hline [18] & annul. slot & 380 & $40 \times 40$ & $2.4,5.2$ & 2 & $2.12,2.75$ & $93 \%, 95 \%$ \\
\hline [19] & annul. slot & 491 & $50 \times 50$ & $\begin{array}{l}2.53,2.92 \\
3.45,4.41\end{array}$ & 3 & $1.2,1.8,3.0,5.0$ & - \\
\hline [20] & rect. slot & 450 & $70 \times 30$ & $\begin{array}{c}1.227,1.381 \\
1.575,2.45\end{array}$ & 3 & $2.4,2.5,2.7$ & - \\
\hline [21] & rect. slot & 675 & $25 \times 27$ & $\begin{array}{c}2.3,5.8 \\
2.3 / 4.5,4.5 / 5.8 \\
\end{array}$ & 3 & $0.7 / 2.5,1.8 / 2.8,0.3,2.5$ & $59 / 67 \%, 85 / 75 \%, 57 \%, 78 \%$ \\
\hline [22] & T-slot & 805 & $23 \times 35$ & 6 to 10.67 & 1.72 & $83 \%$ & \\
\hline [23] & U-slot & 584 & $28 \times 62$ & $\begin{array}{l}1.5,1.6,1.8 \\
1.9,2.24\end{array}$ & 4 & 2.5 & $78 \%$ \\
\hline Prop. & $\begin{array}{l}\text { Conc. Pent. } \\
\text { slot }\end{array}$ & 347 & $60 \times 120$ & $\begin{array}{l}1.32 \sim 1.49 \\
1.76 \sim 5.2\end{array}$ & 2 & $\begin{array}{c}4.5,4.45,4.08,3.98,3.8 \\
3.12,2.78,1.91,0.5\end{array}$ & $\begin{array}{c}75 \%, 68 \%, 71 \%, 79 \%, 81 \% \\
60 \%, 56 \%, 51 \%, 40 \%\end{array}$ \\
\hline
\end{tabular}

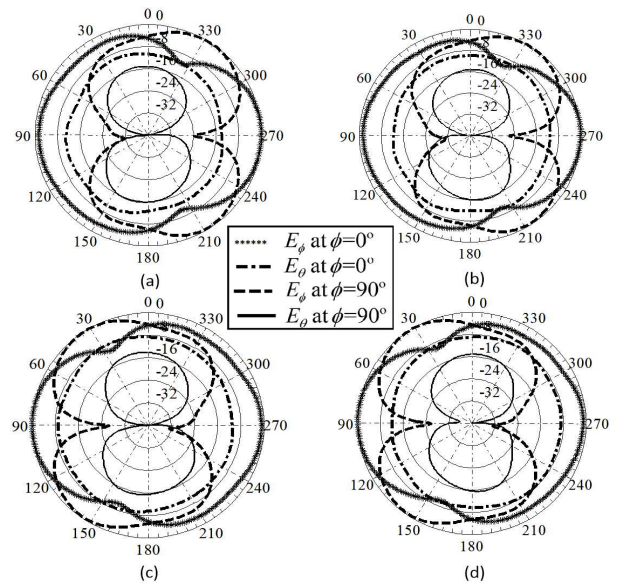

Figure 7: Measured normalized $\mathrm{E}_{\theta}$ and $\mathrm{E}_{\phi}$ at $2.65 \mathrm{GHz}$ (a) Ant-1 (b) Ant-2 (c) Ant-3 (d) Ant-4.

The simulated and measured ECC values for different bands are given in Table III. Good MIMO performance is observed as the ECC values are well below the threshold value.

Another important parameter for MIMO operation characterization is the TARC [24]. The TARC curves were obtained by exciting one port at $1 \mathrm{e}^{j 0}$ with zero phase while the phases of the other ports were varied to different values while keeping the same magnitude of excitations. The excitation phases used were chosen from $0^{\circ}, 30^{\circ}$, $60^{\circ}, 90^{\circ}, 150^{\circ}, 180^{\circ}, 240^{\circ}$ and $300^{\circ}$ for all four ports (P-1, P-2, P-3, $\mathrm{P}-4)$ to form 6-possible cases. The TARC curves describe the effective $\mathrm{BW}$ of the MIMO antenna system. It is clear that the operating BW

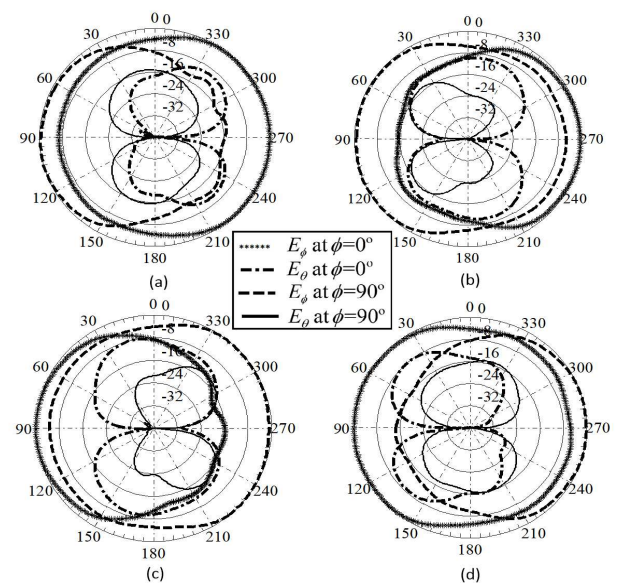

Figure 8: Measured normalized $\mathrm{E}_{\theta}$ and $\mathrm{E}_{\phi}$ at $5.0 \mathrm{GHz}$ (a) Ant-1 (b) Ant-2 (c) Ant-3 (d) Ant-4.

of the proposed MIMO antenna system is robust and not severely affected by phase changes in the excitation of the other ports. The cases considered were: Case- $1: \mathrm{P}-1=0^{\circ} ; \mathrm{P}-2=0^{\circ} ; \mathrm{P}-3=30^{\circ} ; \mathrm{P}-4=60^{\circ}$

Case- $2: \mathrm{P}-1=0^{\circ} ; \mathrm{P}-2=30^{\circ} ; \mathrm{P}-3=90^{\circ} ; \mathrm{P}-4=150^{\circ}$

Case- $3: \mathrm{P}-1=0^{\circ} ; \mathrm{P}-2=60^{\circ} ; \mathrm{P}-3=150^{\circ} ; \mathrm{P}-4=180^{\circ}$

Case-4:P-1 $=0^{\circ} ; \mathrm{P}-2=60^{\circ} ; \mathrm{P}-3=90^{\circ} ; \mathrm{P}-4=180^{\circ}$

Case-5:P-1 $=0^{\circ} ; \mathrm{P}-2=90^{\circ} ; \mathrm{P}-3=150^{\circ} ; \mathrm{P}-4=240^{\circ}$

Case-6:P- $1=0^{\circ} ; \mathrm{P}-2=30^{\circ} ; \mathrm{P}-3=240^{\circ} ; \mathrm{P}-4=300^{\circ}$

From Fig. 9, it can be seen that results for the first four cases obtained are good in the low band of $1.46 \mathrm{GHz}$ for $C=0.9 \mathrm{pF}$; however, the performance is degraded a little for Case- 5 and Case- 6 . For the 
other two higher bands, there is a slight variation in resonance curves and thus the operating BW obtained is quite robust and not severely affected by phase changes in the excitation of the other ports. Hence, good TARC curves are obtained at the higher two bands as shown in Fig. 9 as well as Case-1 to Case-4 at the lower frequency band of $1.46 \mathrm{GHz}$.

Table III: Simulated and measured ECC

\begin{tabular}{|l|l|l|l|l|l|l|l|}
\hline \multicolumn{4}{|c|}{ Simulated ECC } & \multicolumn{4}{c|}{ Measured ECC } \\
\hline $\mathrm{f}_{\text {sim }}$ & $\mathrm{ECC}_{12}$ & $\mathrm{ECC}_{13}$ & $\mathrm{ECC}_{14}$ & $\mathrm{f}_{\text {mea }}$ & $\mathrm{ECC}_{12}$ & $\mathrm{ECC}_{13}$ & $\mathrm{ECC}_{14}$ \\
\hline 4.94 & 0.009 & 0.093 & 0.025 & 4.88 & 0.079 & 0.053 & 0.045 \\
\hline 3.98 & 0.035 & 0.148 & 0.035 & 4.04 & 0.061 & 0.131 & 0.014 \\
\hline 3.4 & 0.062 & 0.186 & 0.075 & 3.38 & 0.013 & 0.123 & 0.022 \\
\hline 3.12 & 0.11 & 0.16 & 0.036 & 3.14 & 0.045 & 0.114 & 0.098 \\
\hline 2.98 & 0.095 & 0.171 & 0.091 & 2.98 & 0.082 & 0.081 & 0.038 \\
\hline 2.65 & 0.135 & 0.012 & 0.083 & 2.66 & 0.098 & 0.0231 & 0.074 \\
\hline 2.24 & 0.162 & 0.054 & 0.086 & 2.45 & 0.152 & 0.087 & 0.022 \\
\hline 1.86 & 0.025 & 0.036 & 0.0182 & 1.88 & 0.032 & 0.044 & 0.0218 \\
\hline 1.46 & 0.095 & 0.171 & 0.091 & 1.47 & 0.082 & 0.081 & 0.038 \\
\hline 1.40 & 0.185 & 0.124 & 0.0125 & 1.41 & 0.175 & 0.098 & 0.0235 \\
\hline 1.35 & 0.092 & 0.025 & 0.148 & 1.36 & 0.096 & 0.042 & 0.168 \\
\hline
\end{tabular}

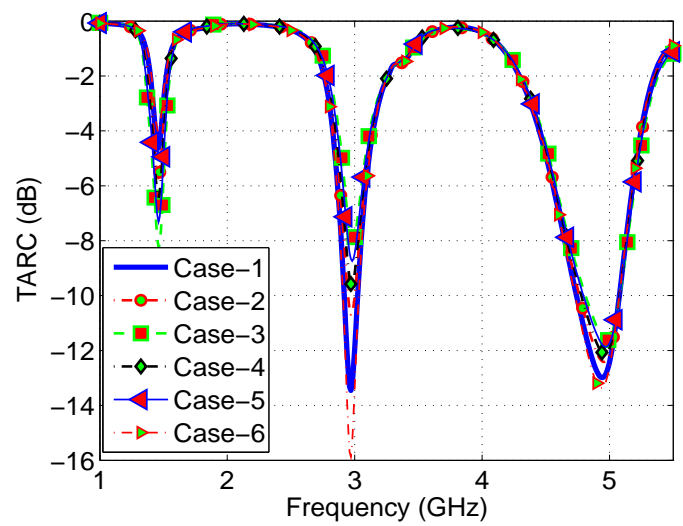

Figure 9: TARC curves for the proposed MIMO antenna system at $\mathrm{C}=0.9 \mathrm{pF}$.

\section{CONCLUSIONS}

In this paper, a compact concentric pentagonal slot-line based 4-element frequency reconfigurable MIMO antenna system is presented. The proposed tir-band antenna system was made ultra-wide tunable using varactor diodes. A smooth variation of resonance frequencies were observed from $1.32 \sim 1.49 \mathrm{GHz}$ and $1.75 \sim 5.2 \mathrm{GHz}$ with tri-band operation. The antenna was fabricated on an FR4 substrate with board of $60 \times 120 \times 1.56 \mathrm{~mm}^{3}$. All the antenna elements were planar in structure that were etched out from the GND plane. The proposed antenna design is well suited for CR platforms in wireless handheld devices and mobile terminals. The proposed antenna design was covering several well-known frequency bands. The antenna was characterized for MIMO performance metrics like ECC and TARC curves. Good MIMO performance metrics are observed.

\section{ACKNOWLEDGMENT}

This work is supported in part by the Deanship of Scientific Research (DSR) at King Fahd University of Petroleum and Minerals (KFUPM) under project number KAUST002 and and in part by the King Abdullah University of Science and Technology (KAUST) Office of Sponsored Research (OSR) under Award No. OSR 2016KKI-2899.

\section{REFERENCES}

[1] J. Mitola, "Cognitive radio architecture evolution," Proceedings of the IEEE, vol. 97, no. 4, pp. 626-641, 2009.

[2] P. Steenkiste, D. Sicker, G. Minden, and D. Raychaudhuri, "Future directions in cognitive radio network research," in NSF workshop report, vol. 4, no. 1, 2009, pp. 1-2.

[3] N. Behdad and K. Sarabandi, "Dual-band reconfigurable antenna with a very wide tunability range," IEEE Trans. Antennas Propag., vol. 54 no. 2, pp. 409-416, 2006.

[4] Z. Hu, C. Song, J. Kelly, P. Hall, and P. Gardner, "Wide tunable dualband reconfigurable antenna," Electronics letters, vol. 45, no. 22, pp. 1109-1110, 2009.

[5] Y. Yu, J. Xiong, H. Li, and S. He, "An electrically small frequency reconfigurable antenna with a wide tuning range," IEEE Antennas Wireless Propag. Lett., vol. 10, pp. 103-106, 2011.

[6] L. Xing, Y. Huang, Q. Xu, and S. Aljaafreh, "A transparent dielectricloaded reconfigurable antenna with a wide tuning range," IEEE Antennas Wireless Propag. Lett., vol. 15, pp. 1630-1633, 2016.

[7] M. Gholamrezaei, F. Geran et al., "Completely independent multi-ultra wideband and multi-dual-band frequency reconfigurable annular sector slot antenna (fr-assa)," IEEE Trans. Antennas Propag., 2016.

[8] T. Li, H. Zhai, L. Li, and C. Liang, "Frequency-reconfigurable bow-tie antenna with a wide tuning range," IEEE Antennas Wireless Propag. Lett., vol. 13, pp. 1549-1552, 2014.

[9] Y. Cao, S. W. Cheung, and T. I. Yuk, "Frequency-reconfigurable multiple-input-multiple-output monopole antenna with wide-continuous tuning range," IET Microwaves, Antennas \& Propagation, vol. 10, no. 12 , pp. 1322-1331, 2016.

[10] Z. H. Hu, P. S. Hall, P. Gardner, and Y. Nechayev, "Wide tunable balanced antenna for mobile terminals and its potential for mimo applications," in Antennas and Propagation Conference (LAPC), 2011 Loughborough. IEEE, 2011, pp. 1-4.

[11] R. Hussain and M. S. Sharawi, "Wide-band frequency agile mimo antenna system with wide tunability range," Microwave and Optical Technology Letters, vol. 58, no. 9, pp. 2276-2280, 2016.

[12] S. Soltani, P. Lotfi, and R. D. Murch, "A port and frequency reconfigurable mimo slot antenna for wlan applications," IEEE Trans. Antennas Propag., vol. 64, no. 4, pp. 1209-1217, 2016.

[13] J.-H. Lim, Z.-J. Jin, C.-W. Song, and T.-Y. Yun, "Simultaneous frequency and isolation reconfigurable mimo pifa using pin diodes," IEEE Trans. Antennas Propag., vol. 60, no. 12, pp. 5939-5946, 2012.

[14] A. N. Kulkarni and S. K. Sharma, "Frequency reconfigurable microstrip loop antenna covering lte bands with mimo implementation and wideband microstrip slot antenna all for portable wireless dtv media player," IEEE Trans. Antennas Propag., vol. 61, no. 2, pp. 964-968, 2013.

[15] H. T. Chattha, M. Nasir, Q. H. Abbasi, Y. Huang, and S. S. Alja' afreh, "Compact low profile dual port single wideband planar inverted-f mimo antenna," IEEE Antenna and Wireless Propagation Letters, vol. 12, pp. 1673-1675, 2005.

[16] H. Li, J. Xiong, Y. Yu, and S. He, "A simple compact reconfigurable slot antenna with a very wide tuning range," IEEE Trans. Antennas Propag., vol. 58, no. 11, pp. 3725-3728, 2010.

[17] "Annular slot-based miniaturized frequency agile mimo antenna system."

[18] B. A. Cetiner, G. R. Crusats, L. Jofre, and N. Biyikli, "Rf mems integrated frequency reconfigurable annular slot antenna," IEEE Trans. Antennas Propag., vol. 58, no. 3, pp. 626-632, 2010.

[19] C.-Y.-D. Sim, T.-Y. Han, and Y.-J. Liao, "A frequency reconfigurable half annular ring slot antenna design," IEEE Trans. Antennas Propag., vol. 62 , no. 6, pp. 3428-3431, 2014.

[20] C. Hung and T. Chiu, "Dual-band reconfigurable antenna design using slot-line with branch edge," IEEE Trans. Antennas Propag., vol. 63, no. 2 , pp. $508-516,2015$.

[21] L. Han, C. Wang, X. Chen, and W. Zhang, "Compact frequencyreconfigurable slot antenna for wireless applications," IEEE Antennas Wireless Propag. Lett., vol. 15, pp. 1795-1798, 2016.

[22] L. Pazin and Y. Leviatan, "Reconfigurable rotated-t slot antenna for cognitive radio systems," IEEE Trans. Antennas Propag., vol. 62, no. 5, pp. 2382-2387, 2014

[23] S. Sharma and C. C. Tripathi, "Frequency reconfigurable u-slot antenna for sdr application," Progress In Electromagnetics Research Letters, vol. 55, pp. 129-136, 2015.

[24] M. S. Sharawi, "Printed mimo antenna engineering," Artech House, 2014. 